\title{
Systems Science and the Art of Interdisciplinary Integration
}

\author{
Sara Green ${ }^{1} \&$ Hanne Andersen ${ }^{2}$
}

\begin{abstract}
Systems sciences address issues that cross-cut any single discipline and benefit from the synergy of combining several approaches. But interdisciplinary integration can be challenging to achieve in practice. Scientists with different disciplinary backgrounds often have different views on what count as good data, good evidence, a good model, or a good explanation. Accordingly, several scholars have reported on challenges encountered in interdisciplinary settings. This chapter outlines how some of the challenges play out in systems biology where disciplinary ideals and domain specific practices sometime collide. We focus on tensions arising due to differences in epistemic standards between modellers with a background in physics or systems engineering, on one hand, and experimenters with a background in molecular biology on the other. We propose that part of the problem of interdisciplinary integration can be understood as the result of unfounded "disciplinary imperialism" on both sides, in which standards from one discipline are uncritically applied to new domains without recognition of other valid or complementary perspectives. We suggest that addressing and explicating the disciplinary background for the different views can help facilitate interdisciplinary collaboration in science as well as serve to improve science education.
\end{abstract}

Keyworkds: Interdisciplinarity; Systems science; Systems biology; Disciplinary imperialism; Epistemic standards

\footnotetext{
${ }^{1}$ Department of Science Education, Section for History and Philosophy of Science, University of Copenhagen, Øster Voldgade 3, 1350 Copenhagen, DK.

Author of correspondence. Email: sara.green@ind.ku.dk, Phone: +45 51331702.

${ }^{2}$ Department of Science Education, Section for History and Philosophy of Science, University of Copenhagen, Øster Voldgade 3, 1350 Copenhagen, DK.
} 
To appear in Systems Research and Behavioral Science, 2019, 36:5. Special issue: ISSS Yearbook.

\section{Introduction}

The term systems sciences comprises a wide variety of approaches and there is not (yet) any firm theoretical foundation or unifying framework for what constitutes a systems approach (Rousseau, 2017; Rousseau, Wilby, Billingham, \& Blachfellner, 2016). However, a central feature of all systems sciences is that aim to solve complex problems that cannot be addressed within the boundaries of any traditional discipline alone. Interdisciplinary approaches carry great potential for exploring new scientific territories and for addressing complex problems in society. Accordingly, interdisciplinary research has been high on the political agenda for many years (Bechtel, 1986; Weingart, 2000). In practice, however, interdisciplinary collaboration is often experienced as difficult and frustrating. One reason may be that institutional or governance structures rarely support research that crosses institutional boundaries. Another is that standards for what counts as "good science" are often domain-specific and hence difficult to integrate.

Much focus has been on minimizing organizational or institutional obstacles (Crow \& Dabars, 2015; Sá, 2008; Wiengart \& Padberg, 2014). In this chapter, we shall instead focus on cognitive obstacles (MacLeod, 2018) and how they are rooted in discipline-specific cognitive constraints. We describe how the tendency to impose explanatory ideals from one field to another presents a challenge to interdisciplinarity, and suggest that increasing the awareness of explanatory ideals can help overcome such challenges. Further, we discuss how traditional science education conveys disciplinary ideals and propose that education can be modified to prepare students for interdisciplinary collaboration. Our analysis will focus on examples from systems biology, which combines experimental approaches within molecular biology with theoretical modelling tools from systems theory, physics, engineering, computer science, and mathematics (Aderem, 2005; Kitano, 2005; Wolkenhauer, 2001).

In systems biology, interdisciplinary integration is considered a prerequisite for addressing biological complexity, since this requires a combination of experimental biology and advanced mathematical and computational modelling. Yet, systems biology is a highly diverse approach where some proponents emphasize the relevance of systems theory to biological research, while others primarily view systems biology as an extension of molecular biology through computational integration of big data from omics fields. Our aim in this chapter is not to provide an account of the characteristics of systems biology (for this purpose, see Green, 2017). However, a division of systems biology approaches into a systems theoretical stream and a pragmatic stream (see O'Malley \& Dupré, 2005) highlights interesting differences in the way systems biologists position themselves in relation to molecular biology and to views on the role of abstract 
To appear in Systems Research and Behavioral Science, 2019, 36:5. Special issue: ISSS Yearbook.

modelling in biology (Calvert, 2010; Calvert \& Fujimura, 2011). Different viewpoints are often based on differences in disciplinary training (in experimental biology or theoretical systems sciences), which may cause tensions when systems biologists with different backgrounds work together in concrete research projects. Our aim is to investigate further what such differences and challenges consist in.

We start with a brief introduction to philosophical accounts that have aimed to describe what constitutes disciplinary and interdisciplinary research activities (Section 2). These accounts provide a conceptual toolkit for explicating, identifying, and discussing disciplinary standards and challenges to interdisciplinary collaboration. To illustrate the proposed approach, we examine case examples from systems biology where ideals in experimental and mathematical research practices collide (Section 3). We shall then discuss various ways in which such a clash between ideals can be overcome though recognition of complementary approaches (Section 4). Finally, we discuss the implications of recent philosophical insights for science education (Section 5), and end with concluding remarks (Section 6).

\section{Conceptual tools from philosophy of science}

Interdisciplinarity is often defined as the integration of multiple disciplinary perspectives, in contrast to multidisciplinarity where the different disciplinary perspectives are merely juxtaposed (Klein, 2010; Lattuca, 2001). ${ }^{3}$ However, it is often left opaque what integration means, and what is being integrated. Integration can refer to many different things such as disciplines, fields, or approaches (Bechtel, 1986; Darden \& Maull, 1977; Griesemer, 2013), problem agendas or explanations (Brigandt, 2013; 2015; Brigandt \& Love', 2012), or methods, models, or data (Leonelli, 2007, 2008; Mitchell, 2002, 2005; O’Malley \& Soyer, 2012). We shall here follow social scientists Jane Calvert and Joan Fujimura (2011) in emphasizing that integration in the context of systems sciences involve both epistemic and social elements.

Systems sciences not only merge data, technologies and methods. They are also shaped by scientists with different kinds of disciplinary expertise who need to collaborate, and in doing so have to bridge between different standards for what is perceived as relevant problems to work on or as high quality solutions (Goddiksen, 2017). As we shall see in Section 3, systems biologists with different disciplinary backgrounds may for instance differ with respect to whether the aim of systems biology should be to formulate fundamental principles of biological organization or detailed mechanistic models and explanations. Such views differ not only in what is considered a useful scientific aim, but also in what

\footnotetext{
${ }^{3}$ This distinction has its root in the first major typology of discipline-crossing approaches that was advanced at an international conference on interdisciplinarity co-sponsored by the OECD and published in a proceeding volume that has become a standard reference for analyses of interdisciplinarity (Apostel, 1972).
} 
To appear in Systems Research and Behavioral Science, 2019, 36:5. Special issue: ISSS Yearbook.

constitutes a good or an acceptable scientific output. We shall argue that different views on such matters often rest on differences in disciplinary training that, again, give rise to different ontological and methodological assumptions.

In order to clarify how standards can clash during interdisciplinary problem solving, it can be instructive to first examine how standards and values are tied to disciplinary problem solving. One of the first philosophers of science to highlight how science is organized was Thomas Kuhn, who emphasized that scientific communities consist of scientists who share values (Kuhn's term for what we shall refer to as epistemic standards) (Kuhn, 1962/1996). He saw science as a process operating within communities of scientists who share a specific set of commitments which he initially termed a "paradigm". On Kuhn's account, the development of a scientific discipline occurs in alternating phases. During normal science, the members of the scientific community adhere to the paradigm in place, that is, they are committed to the puzzles to be solved as well as to the acceptable ways of solving them. However, occasionally, a puzzle may turn out not to be solvable using the tools provided by the paradigm. Such recalcitrant problems Kuhn termed anomalies. As anomalies accumulate, the discipline arrives in a state of crises, where alternatives are developed. One of these alternatives may gradually receive more widespread acceptance until eventually a new consensus has formed among the members of the community on adopting it as the new paradigm. Kuhn argued that the paradigms separated by such a revolutionary divide may in some areas be so different that the relation between the new paradigm and the old cannot be seen simply as one of extension or refinement, but may also include loss of previously accepted knowledge as well as severe communication difficulties between adherents of the old and the new paradigm. Kuhn termed this relation incommensurability.

In has been debated whether the historical development of science, including Kuhn's own example of physics, is characterized by such abrupt changes, or incommensurable paradigms (Brush, 2000). Specifically, Kuhn's phase model of the development of science seems not to fit well with the biological disciplines (O'Malley \& Boucher, 2005). At the same time, the term paradigm has often been used by systems biologists to signal a conceptual change promoted by the 'revolution' of systems biology (Hood \& Flores, 2012). We shall here not go into the debate on whether the emergence of systems biology constitutes a scientific revolution (for a discussion of this issue, see Calvert \& Fujimura, 2011). Instead, by drawing on Kuhn's description of the different kinds of commitments that scientists within a given discipline share, we shall show how these elements of the disciplinary matrix may serve as useful conceptual tools to address differences in disciplinary standards in interdisciplinary settings. Later, we shall 
To appear in Systems Research and Behavioral Science, 2019, 36:5. Special issue: ISSS Yearbook.

return to Kuhn's account of science and describe how science education serves to transmit the elements of the disciplinary matrix from one generation of scientists to the next.

Clarifying and refining the idea of a paradigm, Kuhn introduced the notion of a disciplinary matrix to denote the entire constellation of shared beliefs and techniques (Kuhn, 1962/1996): First, symbolic generalizations are the formal laws or principles agreed upon by members of a research community, such as Newton's second law or Ohm's law in physics. Second, metaphysical assumptions are the basic and shared assumptions about the nature of the research objects, e.g., that that physical processes arise due to interactions of matter and force that can be described mechanistically. Third, values are criteria for good science or virtues of theories or models such as simplicity, consistency, or accuracy. Finally, exemplars are canonical problem solutions that illustrate the applicability of symbolic generalizations or examples of good explanations. Kuhn was primarily interested in how individual disciplines develop over time, not in how at some given time different disciplines may relate to each other. However, Kuhn's elements of a disciplinary matrix may still be useful for clarifying how scientists working in an interdisciplinary field like systems biology may adhere to different domain specific standards due to their different disciplinary backgrounds. Challenges to interdisciplinary integration in systems biology may, at least in part, result from differences in values for good science. For instance, there are different views on whether data-intensive (or data-driven) approaches should be considered science at all, and whether Occam's razor is still a useful principle in systems biology (Gross, 2017; Kolodkin \& Westerhoff, 2011; O’Rourke \& Crowley, 2012). We shall here primarily focus on differences in values concerning good models and explanations, because these often differ among scientists trained in theoretical and empirical disciplines.

In the context of systems biology, discussions on different standards for what constitutes a good model or explanation were stimulated by a provocative paper entitled "Can a biologist fix a radio?", published by the cancer biologist Yuri Lazebnik (2002). Frustrated with the inability to predict and explain cancer, despite heavy investments in this research area, Lazebnik reflects upon potential blind spots and flawed methodologies inherent in current research strategies. He points out how the strategy of molecular biology would not be useful for fixing a radio, as the strategy would amount to crushing down the system into component parts and studying these in isolation. In both cases, he argues, the strategy is likely to fail because the problem may not lie in any specific malfunctioning component but in disturbances to the system-level organization. As a more promising approach, Lazebnik highlights the approach of an engineer who starts out by mapping a wiring diagram of the radio to understand the dynamic principles the system is based on. Lazebnik's paper received much criticism from biologists, not least because of the comparison of 
To appear in Systems Research and Behavioral Science, 2019, 36:5. Special issue: ISSS Yearbook.

problem solving in biology and reverse engineering of a radio. However, the analogy highlights some interesting aspects that can be framed in terms of the elements of a Kuhnian matrix. In the following, we will illustrate this by describing the some of the main components of the disciplinary matrices of both molecular biologists and system theorists and illustrate how differences between them can create challenges for interdisciplinary collaboration. ${ }^{4}$

First, differences in metaphysical assumptions about the research object may lead to different methodological choices, and vice versa. For example, the metaphysical worldview of molecular biology may be described as one where mechanistic details matter and biological complexity prohibits description via simplified laws (see e.g., Machamer et al, 2000; Matthiessen 2017). From their experimental experiences, many biologists have learned that even small changes in molecular compositions or details of protein concentration or folding properties can result in radical changes to functions at the phenotypic level. From their perspective, a careful and detailed examination of the system's components and interactions may therefore be considered more fruitful than to start the investigation from a set of idealized and abstract models, as physicists or engineers are inclined to do. This difference has been documented by interviews by philosophers with biologists and physicists who work together in systems biology projects (Rowbottom 2011; Joaquim, Freire \& El-Hani, 2019). ${ }^{5}$ The interviews show that physicists often use models which appear to be too coarse-grained and idealized to seem useful to the biologists, because these are not directly anchored in causal details of concrete biological systems. The different emphasis on rooting of models in empirical details also result in different views on whether computer simulations can count as experimental interventions (see also (Carusi 2012; 2014) for similar observations and a discussion of the model-experiment distinction).

This difference with respect to metaphysical assumptions is closely tied to differences with respect to the role played by symbolic generalizations. Such generalizations only play a minor role in molecular biology, in

\footnotetext{
${ }^{4}$ The following will, too, be a coarse-grained picture that does not give justice to the nuances and diversity of worldviews held by systems biologists with different training. But we hope that our analysis provides a framework for understanding some of the tensions and challenges observed and exemplified in empirical studies of interdisciplinary collaboration in systems biology (see below and Section 3).

${ }^{5}$ The physicist Wilson Poon frames the view of biologists vs. physicists nicely in the following quote: "Chemical details are everything. Ultimately, no two proteins are alike. A 'point mutation' changing one residue (=amino acid) in a 100-residue protein can make the difference between life and death. Such specificity contrasts starkly with the instinct of the soft condensed matter physicist to focus on generic features by coarse graining - ignoring enough details until similarities begin to emerge" (Poon 2006: 2, quoted in Rowbottom 2011, who offers a set of illuminating interpretations of this and similar examples).
} 
To appear in Systems Research and Behavioral Science, 2019, 36:5. Special issue: ISSS Yearbook.

part because of the complexity, contingency, and diversity of the molecular systems studied, and in part because of the weight on experimental procedures and expertise. While some generalizations are possible, derivation of relevant research results from "first principles" is typically considered impossible due to the contingency of "dirty, unruly living systems" (biologist cited in interview by Calvert and Fujimura 2011, p. 161). In contrast, abstraction from details and application of idealized models is for the physicist or engineer the most promising way to deal with complexity (Rowbottom, 2009; 2011).

Textbook exemplars of molecular biology can be general, such as the regulatory principles underlying the lac operon, protein synthesis, or mechanisms for genetic inheritance. But these are typically presented with reference to concrete material entities and operations such as specific macromolecules (Campbell \& Reece, 2005; Machamer, Darden, \& Craver, 2000). In contrast, the engineering inspired approach to biology is characterized by a striking lack of molecular details. One example is Uri Alon's (2007) textbook An Introduction to Systems Biology. Design Principles of Biological Circuits. Exemplars in Alon's book focus on regulation of gene expression and protein synthesis. But unlike textbooks exemplars in molecular biology on the same topic, engineering-inspired diagrams do not place weight on the molecular characteristics of specific proteins and transcription factors. Rather, these are represented by formal abstractions $(X, Y, Z$, etc), and the organizational relations between these are in focus. Functions are not explained through specific molecular interactions, but through design principles, i.e., through generic control principles such as amplifiers, filters, feedback circuits, bi-stable switching, etc. Rather than a focus on the causal details of any specific system, the book highlights features of biological functioning that are shared with engineered systems (Green, 2015b). Learning about biological functions through systems biology textbooks thus differs in interesting ways from the training of molecular biologists. ${ }^{6}$

Accordingly, the weight on values (in a Kuhnian sense) for good models also differ. Molecular biologists often place high emphasis on accuracy and concrete representation, rather than simplicity and generality based on mathematical abstraction. These values resonate with the worldview of living systems as something that is too diverse and complex to be described solely via physical or chemical principles. Biologists and philosophers of biology have maintained that explanations in biology need not be generalizable to be explanatory, as accounts based on physics had emphasized (c.f., Hempel, 1966; Machamer et al., 2000). That living systems are not amenable to generalizable laws, and that laws do not constitute ideal examples in biology, sometimes collide with the approach of systems scientists trained in

\footnotetext{
${ }^{6}$ In previous work, one of the authors has clarified the explanatory relevance of design principles and how these relate to mechanistic explanations that are more familiar to biologists (Green and Jones, 2016).
} 
To appear in Systems Research and Behavioral Science, 2019, 36:5. Special issue: ISSS Yearbook.

physics or engineering. These scientists would often start with models known from physics, or proceed through identification of dynamic patterns of control that resemble design principles known from engineered systems. Models inspired by engineering and physics are relatively insensitive to causal details of any specific system (Joaquim, Freire \& El-Hani, 2019). Whereas this insensitivity is perceived as an explanatory virtue from the perspective of physicists or engineers, biologists may view this as a shortcoming of formal models.

The valued aspects of exemplars in the two contexts are described by an engineer, interviewed by Calvert and Fujimura (2011, p. 160), as follows: "engineers are always looking for general principles, whereas biologists are more interested in outliers". Again, the preference for more or less abstract models or principles can partly be understood as a difference in standards of quality (or explanation), but may also rest on profound differences in the ontological assumptions about living systems. It amounts to different views on whether "biology is written in the language of mathematics" (Calvert \& Fujimura 2011, 160). If not, contingencies and particularities in biological evolution may make biology "nomically inhibited" (Burian, Richardson, \& Van der Steen, 1996). ${ }^{7}$ From this perspective, a consequence of the complexity, variation and context-dependency of biological functions is that the life sciences require a different explanatory framework than physics. Thus, complexity and the necessity of accounting for details is for the molecular biologist important features that support the disciplinary autonomy of biology.

Although these differences may be described as differences in "standards of quality", they also regard fundamental assumptions about nature - and may also influence the more specific framing of relevant research questions (standards of relevance). If, following Lazebnik, cancer is not a disease of any specific molecular components or pathways, searching for a malfunctioning component is akin to searching for a cat in darkness that is not even there (Lazebnik 2002). On the other hand, if the complexity of biological system makes it impossible to arrive at simple, general laws governing the system, or if biological and engineered systems turn out to be very different in terms of functional organization, then the search for fundamental principles may also be viewed as futile (Calvert \& Fujimura 2011). Our aim here is not to evaluate what is most useful, but primarily to highlight that each scientific worldview has characteristic implications - understood as relative merits and limitations.

\footnotetext{
7 The notion of nomical inhibition is based on the view that no law is universally applicable to the biological domain due to the diversity and contingency of living systems (see also Mayr, 2005). From this perspective, biology is characterized by numerous interconnected theories and explanatory examples that acquire meaning in relation to specific biological contexts, rather than in relation to fundamental laws (Burian et al., 1996).
} 
To appear in Systems Research and Behavioral Science, 2019, 36:5. Special issue: ISSS Yearbook.

The differences outlined so far with respect to differences in standards of relevance and quality, as well as with respect to components of Kuhn's disciplinary matrix, will be further illustrated in the following. As source material we shall combine an analysis of published scientific papers, interactions with and observations of practicing systems biologists, as well as recent empirical work in philosophy of science and social science investigating interdisciplinary collaboration in systems biology. We shall begin with challenges that may be explained in terms of different explanatory standards (or ideals concerning exemplars) (3), and then proceed with a clarification of the problem with a suggestion of potential ways of overcoming the challenges (4). On this basis, Section 5 will discuss the implications of cognitive obstacles for science education.

\section{Challenges to explanatory integration}

Challenges to integrate abstract systems-theoretical or engineering principles and experimental biology are identifiable throughout the history and of systems biology and its precursor approaches. Attempts to apply systems theoretical principles to biology were initiated already from the 1930ies through the pioneering works of Ludwig von Bertalanffy, Paul Weiss, Nicolas Rashevsky, and onwards by Robert Rosen, Mihajlo Mesarovic, Michael Savageau, and many others (for historical overviews, see Drack \& Wolkenhauer, 2011; Green \& Wolkenhauer, 2013). But a unified or experimentally integrated systems theoretical approach to biology has not yet materialized (Rousseau, 2017; Rousseau et al., 2016). One reason for the lack of uptake may be due to collision of explanatory standards.

Already in the 1930ies, Rashevsky addressed the problem of scepticism among biologists of the utility of mathematics (Abraham, 2004; Kjeldsen, 2017; Rashevsky, 1938a, 1938b). While Rashevsky was aiming to develop a mathematical biology, akin to mathematical physics, biology was considered as being too complicated or complex to be submissive to mathematical analysis. Thus, experimental biologists viewed the top-down approach of mathematicians or physicists as disrespectful to the complexity of nature. Ironically, however, systems theoreticians or mathematical biophysicists viewed the attempt to fully grasp this complexity via experiments and "realistic" complex models as equally unrealistic or arrogant. Rashevsky for instance sarcastically remarks that: "Only a superman could grasp mathematically at once all the complexity of a real thing. We ordinary mortals must be more modest and approach reality asymptotically, by gradual approximation" (Rashevsky, 1938a, p. 1; see also Shmailov, 2016). ${ }^{8}$

\footnotetext{
${ }^{8}$ What comes before this quote gives a view of how Rashevsky felt a need to "educate" the biologists: "The objection may be raised against such an approach, because systems have no connection to reality; and therefore any conclusions drawn about such idealized systems cannot be applied to real ones. Yet this is exactly what has been, and always is, done in physics. The physicist goes on studying mathematically, in detail, such nonreal things as "material
} 
To appear in Systems Research and Behavioral Science, 2019, 36:5. Special issue: ISSS Yearbook.

Many contemporary systems biologists have experienced similar scepticism concerning the utility of abstract mathematical modelling. Rowbottom reports on how a model for a systems biology project, developed by a physicist, was accused of "employing the physicist's typical spherical cow approximation". (Rowbottom 2011, p. 147). The analogy refers to a joke in which a dairy farmer consults a physicist for advice on optimization of milk production, and the model of the physicist begins with an assumption of a spherical cow. The point is here that biologists often view the abstract models developed by mathematicians, engineers of physicists as being too idealized to be applicable in biology (see also Doyle 2001). Similarly, one of the scholars who has played important roles in the development of Biochemical Systems Theory, Eberhard Voit at Georgia Tech, explains how new ideas for an applied mathematics for biology was hampered not only resistance practicing biologists but also from "pure mathematicians" (Voit, 2017). The mathematical and biological world was viewed as two different spheres. During Voit's time as a post.doc. with Michael Savageau at University of Michigan, they experienced having a funding proposal rejected by the program director of the NSF math division with the comment that "math had been around for a couple of 1000 years and that it was not very likely that someone at a medical school would come up with something new" (Voit 2017, p. 248). As Voit remarks, the idea that biology is too complicated to use math must has been turned upside down in modern systems biology, in the sense that "biology may be too complicated not to use math" (ibid). Still, it is a matter of controversy which types of mathematical frameworks are most applicable in biology for addressing various explanatory questions.

One controversial issue is the prospects and limitations of applications of dynamical systems theory to understand genotype-phenotype relations and to guide research within stem cell biology, developmental biology, and cancer research. Dynamical systems theory has been proposed as "a framework of first principles of regulatory dynamics" (Huang, 2009, p. 546) that makes up the basis for constructing gene regulatory networks (GRNs). Via simple abstract network models, consisting of a finite set of elements (nodes representing products of gene expression) and dynamic interactions between these, the framework provides a way of linking levels of gene expression over time as a set of associated (and mutually constrained) state variables. A key idea is that the dynamics of cells, such as cell differentiation and bistable expression states, can be formally expressed as trajectories towards attractors and bifurcation dynamics. The hope is that application of this framework with time will turn biology into a more

points," "absolutely rigid bodies" "ideal fluids," and so on. There are no such things as those in nature. Yet the physicist not only studies them but applies his conclusions to real things. And behold! Such an application leads to practical results - at least within certain limits. This is because within these limits the real things have common properties with the fictitious idealized ones!" (Rashevsky 1938a, p. 1). 
To appear in Systems Research and Behavioral Science, 2019, 36:5. Special issue: ISSS Yearbook.

quantitative and predictive discipline (Huang, 2011b; Kaneko, 2011). Yet, the proposal of this highly formal approach has often been met with resistance from experimental biologists, or ignored completely (Fagan, 2010,$2012 ;$ 2016). As we shall describe below, one important reason for this is the differences in explanatory standards and how this is reflected in the rhetorical style of publications from the different disciplines (see also Green, Fagan, \& Jaeger, 2015).

In molecular biology, exemplar models and explanations are typically based on empirical experiments. Publications in this field therefore typically contain mechanistic diagrams that track the causal interactions of a small set of specified molecular components in detail. To the proponents of the dynamical systems (DS) framework, however, such models are often considered as preliminary "ad hoc schemes of causal arrows" as they are not "anchored in mathematical principles" (Huang, 2011a, p. 2249). ${ }^{9}$ I contrast, DS theorists aim for universal models and explanations. Consequently, their publications highlight how dynamical systems theory provides a conceptual framework for describing gene regulation through a set of general dynamic principles. This view may however miss out on why experimental biologists are more often interested in developing mechanistic models of how specific systems work. As highlighted in Section 2, biologists may not consider the explanatory power of such models as hampered by the lack of generality. Instead, models are evaluated on the basis of accurate representation of concrete real-world components (Bechtel \& Richardson, 1993). Thus, experimental biologists place different emphasis on the virtues of good scientific explanations. To understand the scientific controversy, it is thus important to recognize that DS theorists and experimental biologists have different epistemic standards for what count as good models or explanations, and may perhaps also be addressing different explanatory questions.

Experimenters are often aiming to answer questions about how a particular biological system works, and may accordingly not consider it useful to develop a mathematical model that illustrate (or subsume) the causal dynamics in a more formal way. But the DS theorists aim for such models because these address a different question of why a wide range stem cell phenomena are possible and why causally diverse systems display characteristic patterns (Furusawa \& Kaneko, 2012; see also Green \& Jones, 2016; Joaquim, Freire \& El-Hani, 2019). If the latter aim is not recognized by experimental biologists, abstract models may seem insufficient in that they only provide "how-possibly" explanations and not explain "how-actually" the system achieves a given function or behaviour (Craver, 2007; Fagan, 2016). The dynamical systems models,

\footnotetext{
${ }^{9}$ Similarly, Furusawa and Kaneko (2012, p. 398) argue that: “[C]omplicated mechanisms... can neither identify the essential features of stem cells nor extract universal features that are present in all stem cell systems. By contrast, we start with a simple model containing only the essential features of biological systems, and with this we attempt to capture the universal behaviour exhibited by all cell societies" (see also Fagan, 2016).
} 
To appear in Systems Research and Behavioral Science, 2019, 36:5. Special issue: ISSS Yearbook.

in their view, primarily restate in a formal way what they already know from their experiments. This view of abstract models among experimenters seems to be common. Based on ethnographic studies of and interviews with modellers and experimenters in integrative systems biology, philosophers of science Miles MacLeod and Nancy Nersessian report that "experimenters often interpret models as just reproductions of old data - or as 'tautologies' that can offer no new insight" (MacLeod \& Nersessian 2014, p. 233-234, our emphasis). Similarly, an interviewee in a project on interdisciplinary collaboration within social science explained that "when physicists produce a model that matches existing data, biologists will often be unimpressed because what is predicted is what is already known to them" (Calvert \& Fujimura, 2011, p. 159).

For experimental biologists to embrace the potential of (dynamical) systems theory, it is important that modellers clarify what their models are intended to help explain. In many cases, explaining any particular system is not the only or even the main target of abstract modelling efforts. To more theoretically inclined modellers, specific systems are primarily interesting insofar as they instantiate a more general type of dynamic principle that can help us understand how specific functions are possible at all or that could perhaps be reproduced in synthetic organisms (Green, 2017). For these purposes, going into too much detail about specific systems can in hinder understanding. When zooming in on the details, there is a risk of "missing the wood", i.e., the dynamic and organizational features that unite characteristic types of systems (e.g., Hofmeyr, 2017; Wolkenhauer, 2017). Whereas this explanatory aim may be self-evident to theoretically inclined modellers, it may be opaque or foreign to experimental biologists, which is why a clarification of aims and standards may be useful for collaborations.

The interest in more general organizational principles or laws is common to various streams within systems theory that draw on engineering or physics. From this perspective, the biologists' approach may be perceived as an obsession with details, leading to an idiosyncratic and "essentially atomistic paradigm" (Rowbottom 2011, p. 148). Accordingly, collaborations are often hampered by different views on the relevant level of detail of the analysis, or by different views on what the ideal research output should be. Similar explanatory discrepancies concerning the possibility of general principles in biology can be observed in the history of evolutionary biology. Whereas neo-Darwinians aimed for selectionist explanations of how specific features have developed, neo-Rationalist (or structuralist) approaches sought to identify general principles that constrain and explain evolutionary trajectories (Smith, 1992). The inheritance of these traditions concerning explanatory aims may result in collaborative challenges in more recent integrative approaches such as EvoDevo and Evolutionary Systems Biology (Green et al., 2015; Griesemer, 2013). 
To appear in Systems Research and Behavioral Science, 2019, 36:5. Special issue: ISSS Yearbook.

In the following section, we propose that part of the problem of interdisciplinary integration can be understood as the result of unfounded "disciplinary imperialism" on both sides, and we point to ways in which tensions could be overcome. Once the paradigm or disciplinary matrices of other fields are better understood, it can be easier to develop joint work that contributes with answers to different - but related research problems. However, not all problems are solved through discussions of explanatory standards. Part of the problem is also the lack of expertise and familiarity with concepts, methods, and techniques in the fields of collaborators. Accordingly, addressing this problem requires different tools than awareness of different epistemic ideals (Section 5).

\section{Reduction, imperialism and suggestions for practical integration}

When disciplines that have different standards of relevance and quality are bought together, scientists from one discipline may attempt to impose their standards on the work of their collaborators who come from other disciplines. Traditionally, such imperialistic tendencies have been associated with theory reduction and the view that special sciences in time would be reducible a more fundamental discipline operating on lower levels (Andersen, 2001a). The notion of fundamentality here refers to a view of disciplines as operating on specific levels (or scales) of organization, and it was envisioned that biology with time could be reduced to chemistry and chemistry to physics (Oppenheim \& Putnam, 1958). The view of physics as a more fundamental science that will eventually account for all other disciplines is sometimes described as physics imperialism (Chang, 2015). An illustrative example is Ernest Rutherford's statement that all science is either physics or stamp collecting. The capacity of physics to reduce theories of other disciplines has been criticized by scientists and philosophers alike, some of which have also questioned Oppenheim and Putnam's "layer-cake" model of science (for an overview, see Brigandt \& Love, 2017). Yet, science imperialism need not be tied to views on reduction of levels of explanation. Recently, philosophers of science have addressed how scientists, regardless of the level of analysis in their field, may act imperialistic in imposing their disciplinary standards on research outside their own discipline (Dupré, 1994; Green et al., 2015; Mäki, 2013).

The notion of disciplinary imperialism is relevant for conceptualizing the problems that may arise when integrating resources from different disciplines in systems biology. Such imperialism may be imperialism of scope when scientists seek to explain phenomena that belong to the perceived domain of another discipline, imperialism in style when they attempt to transfer their techniques or standards of inquiry and communication to other disciplines, imperialism of standing when they attempt to increase their academic 
To appear in Systems Research and Behavioral Science, 2019, 36:5. Special issue: ISSS Yearbook.

prestige and power on the expense of another discipline (Mäki 2013), or imperialism of explanation (Fagan 2016; Green, Fagan \& Jaeger 2015). From the perspective of experimental biologists, proponents of systems theory are often seen as attempting to "conquer" the territory of biology. But, as we shall argue below, experimenters may also have complementary "imperialistic tendencies" that need to be confronted to overcome domain-specific cognitive obstacles in interdisciplinary setting.

Sections 2 and 3 pointed to how explanatory standards in biology may differ from disciplines that favour abstract laws and generalizable formal explanations. The scarcity of formal principles or laws in biology has sometimes been seen as a feature that renders biology "less scientific" that the "hard sciences". For instance, Rachevsky expressed with regret that while "physics developed into a rational mathematical science, biology, as a younger science, must still, on the whole be classified as a purely empirical descriptive one" (Rashevsky, 1938a). Biology is thus viewed as an immature science that needs to follow the example of physics and apply what Rashevsky called the physico-mathematical method: "This use of abstract conceptions in the beginning is the characteristic of the physico-mathematical method. Violation of this rule, and all attempts to start with actual cases in all their complexity result in failure and have contributed to a sceptical attitude towards mathematical methods" (Rashevsky, 1935, p. 528, original emphasis). More recently, some systems theoretical proponents have argued that the mechanistic and pathway-oriented view has to be replaced with a theoretical approach that derives biological explanations from mathematical "first principles" (e.g., Huang 2009). Recall also that exemplars of molecular biology, mechanistic diagrams and explanations, were considered as hand-waiving metaphors or ad hoc interpretations (Section 3). On this basis, experimenters may rightly feel that their contributions are underestimated and downgraded to "stamp collecting". ${ }^{10}$ Meanwhile, it is not obvious to experimenters that theoretical approaches offer any viable alternative or even a useful addition to their accounts, given the research questions they are interested in.

The dismissive reaction from experimenters, however, may also points to the necessity of broadening the conception of disciplinary imperialism beyond a fundamentalist or physicalist approach. The lack of appreciation of systems-theoretical approaches from the experimenters' side may also expose unfounded assumptions of what constitute useful models or explanations in biology. If it is taken for granted that all

\footnotetext{
10 To experimenters, the ideal of systems-theoretical principles may be perceived as a form of mathematical reduction of biological complexity, akin to historical misconceptions of physics as being a model discipline because it provided "harder" covering-law explanations (Hempel, 1966; Hempel \& Oppenheim, 1948). For further discussion of the historical debate between Rashevsky and experimental biologists, see (Kjeldsen, 2017).
} 
To appear in Systems Research and Behavioral Science, 2019, 36:5. Special issue: ISSS Yearbook.

(good) biological explanations have to be detailed causal-mechanistic explanations, then all systems theoretical inputs will be dismissed or downgraded to heuristic tools for mechanistic explanations. To exemplify, we quote a philosopher of science whose view may represent the perspective of many experimenters: "Reasoning using abstract structural analogies is rightly identified as a useful and sometimes powerful heuristic for schema construction, but explanation cannot stop there. Fascination with the apparent autonomy of design should be tempered by a realization that abstract principles bar us from explaining the actual workings of any given system of sufficient complexity" (Matthiessen, 2017, p. 21). ${ }^{11}$ Without dismissing the importance of explanations that describe the actual workings of concrete living systems, the tendency to view such explanations as the only ones relevant to biology may constitute another form of explanatory imperialism. Thus, explanatory imperialism not only includes reductionist attempts to make other disciplines redundant, but also attempts to defend current explanatory territories from influences from other disciplines. Explanatory imperialism, generally understood, is thus the tendency to view the explanatory standards of one's own field as primary or privileged (Green, Fagan \& Jaeger 2015). To facilitate collaboration across disciplines, it can be a useful exercise to identify and question such assumptions.

Understanding the background for disciplinary controversies can clarify why proponents of each field may view the other as "reductionist" or "imperialistic". A further specification of reductionism may also be useful in this context. While systems biologists generally position themselves in opposition to reductionism, and embrace some form of holism, it is not always clear what reductionism or holism refer to. Specifically, it is interesting to note that the discrepancy between those trained in molecular biology and systems engineering can be framed as oppositions to two different types of reductionism. Systems engineers criticize molecular reductionism in molecular biology, i.e., the idea that systems functions can be understood through studies of molecular components and component operations in isolation from the context of the system as a whole. ${ }^{12}$ Meanwhile, experimental biologists trained in molecular biology often oppose what they view as physical or mathematical reductionism (or more generally theory reduction), i.e., reduction of one disciplinary framework to a more fundamental discipline. (Brigandt \& Love, 2017). The

\footnotetext{
11 The quoted passage is from a paper discussing the explanatory value of integral feedback control as a design principle that explains the dynamics of robust adaptation in bacterial chemotaxis. Matthiessen further argues that: "If identifying the dependence of robust adaptation on integral control counts as explanation, then we must say that bacterial chemotaxis and thermostats are here explained in the same way. Such is the generality of design explanation [...], but surely we want our sciences to employ a form of explanation that enables us to distinguish between these systems. This can only occur if models are understood to be based on and correspond to the actual organization of such systems" (Matthiessen, 2017, p. 16, our emphasis).

12 This anti-reductionist view can be further distinguished into responses to so-called "modular reductionism" and “bottom-up reductionism” (Gross \& Green, 2017).
} 
To appear in Systems Research and Behavioral Science, 2019, 36:5. Special issue: ISSS Yearbook.

concern is that "the kinds of tacit knowledge that biologists have gained from years of learning about the details of historical development could disappear when top-down physical, engineering and computer science models are used to select and shape biological data" (Calvert \& Fujimura 2011, p. 159). It is therefore relevant to examine the merits and limitations of both types of reductionism and imperialistic views.

Explanatory imperialism may be partly overcome through greater attention to the assumptions underlying explanatory standards and recognition of the fact that these could be thought differently. Recognition of different explanatory aims and practices can lead to greater acceptance of complementary methods and perspectives. Examining the variety of research practices in the life sciences, philosophers of science have recently reached what looks like a consensus on explanatory pluralism (Braillard \& Malaterre, 2015; Mitchell, 2005). In the context of systems biology, it has been argued that the quests for mechanistic explanations and design principles are complementary in the sense that the former explain how particular systems work, while the latter provide a categorization of types of systems behaviours that are applicable to a wider range of causal systems (Green \& Wolkenhauer, 2013). The latter can thus provide an understanding not only of how specific behaviours are causally produced but also why many different systems rely on similar organizational principles (Braillard, 2010; Green, 2015b).

It could be objected that although accepting the co-existence of different explanatory projects may help avoid some scientific controversies, still this does not really provide a step towards interdisciplinary integration. If modellers and experimenters are pursuing different epistemic aims and standards, what are the prospects of joining disciplinary resources toward interdisciplinary problem solutions? One attempt to answer this question could focus on the possibilities of developing integrative projects. Experimentalists are increasingly faced with datasets that cannot be analysed by informal models alone, while modelers increasingly appreciate the need to demonstrate the empirical relevance of mathematical and computational approaches (Doyle, 2001).

The notion of a boundary object has been introduced as a term for entities (e.g., an abstract model or concrete phenomenon) that bridge between disciplinary boundaries (Star \& Griesemer, 1989). Boundary objects are characterized as phenomena that are open for multiple interpretations and developments, while also providing a joint core or focus of cross-, trans- or interdisciplinary research activities. In the context of systems biology, computational network approaches offers a way to turn Waddington's notion of an epigenetic landscape (modelled as a phase space of cell states) is a candidate for a boundary object (Fagan 2012). The epigenetic landscape serves as conceptual framework with the potential to integrate 
To appear in Systems Research and Behavioral Science, 2019, 36:5. Special issue: ISSS Yearbook.

mathematical modelling based on dynamical systems theory and experimental manipulation of cell development (Fagan, 2017). In this context, theoretical approaches can benefit from experimental resources that gives their abstract models empirical (or biological) grounding, while dynamical systems theory in time may allow for prediction of developmental trajectories based on quantitative modelling (Green et al., 2015a; 2015b).

Similarly, experimenters and systems theoretical approaches may take a joint interest in the identification of biological design principles, despite different explanatory projects. Although design principles may not be the ultimate explanatory aim for experimenters, design principles can be useful as heuristic tools in the format of computational templates or mechanistic schemas (Matthiessen 2017). Moreover, experimental interpretation and scrutiny of such principles can provide a useful corrective to "generalist" engineering approaches. For instance, the discovery of a set of simple and highly frequent regulatory "network motifs" (Alon, 2007) has been followed by scientific and philosophical debates on the potentials and shortcoming of simple abstract models in more complex biological contexts (Green, 2015a; Isalan et al., 2008). A final example is the dependence of shared mathematical tools by both modellers and experimenters, such as Michaelis-Menten representations of biochemical kinetics. Ordinary and partial differential equations provide a connection between abstract modelling within the frameworks of Control Theory and Biochemical Systems Theory and representation of interactions that can be manipulated experimentally (Doyle 2001). In such contexts, inputs from different disciplines can give rise to synergistic effects and iterative corrections without a requirement that collaborators may always pursue identical epistemic aims (MacLeod \& Nersessian, 2014).

Indeed, the degree of specialization in modern science often means that it would be impossible for one individual to develop expertise in all required specializations to address a complex problem. But complete coherence of epistemic aims and expertise may not be a requirement for interdisciplinary integration. A greater awareness of different disciplinary standards and types of expertise can sometimes facilitate sufficient "cognitive flexibility" to also contribute to other research aims than those primary to one's own field. Yet, scientists need to be able to communicate sufficiently to combine their partial contributions (Andersen, 2016; Petrie, 1976). In order to do so, they do not need to acquire full contributory expertise in more than one discipline, but instead what the sociologists Harry Collins and Robert Evans (2002) call interactional expertise (see also Goddiksen, 2014a, for important criticism and clarification of these notions of expertise). Interactional expertise allows for engagement with research within other disciplinary settings without being able to contribute as scientists within that field. Interestingly, results of a recent 
To appear in Systems Research and Behavioral Science, 2019, 36:5. Special issue: ISSS Yearbook.

philosophical project suggest that small "philosophical interventions" in collaborative research practices within Integrative Systems Biology can lead to big improvements in terms of the development of interactive expertise (MacLeod \& Nersessian, 2014). ${ }^{13}$

Interviews with practicing scientists can reveal insights into how collaborators from different fields are viewed (Joaquim, Freire \& El-Hani, 2019). Experimenters often portray modellers as being experimentally or biologically naive, in that they may be asking for data that is not possible to experimentally create or would take much longer than the use of data would be able to justify (MacLeod, 2018). Meanwhile, modellers often experience that the experimenters are unable to understand the details and significance of their models, which from an experimenter's perspective may draw on mathematical or computational strategies. While such problems can partly be framed as one of differences in explanatory standards, they also concern questions about the consequences of large differences in the degree of mathematical and experimental training, respectively. This leads to a discussion of the implications of insights to interdisciplinary challenges for science education.

\section{Implications for interdisciplinary education}

We initiated the analysis of disciplinary standards by drawing on Kuhn's account of normal science. In the following, we shall return to Kuhn's account of science and the role that science education plays in this account. As Kuhn saw science education at the university level, it serves to convey the elements of the disciplinary matrix to the students of a discipline through exemplary problems and their concrete solutions. In this process, a set of concrete problems and exemplary problem solutions are displayed to the students, who are then asked to mimic these exemplars in solving additional problems of the same type. This process constitutes a dogmatic initiation into the discipline's problem-solving tradition, and Kuhn argued that this dogmatism was beneficial for science. First, he saw it as beneficial for normal science because the confident and continuous use of accepted concepts, theories and methods increases the effectiveness and efficiency with which the problems within a given discipline are solved. Second, Kuhn also argued that this

\footnotetext{
13 MacLeod and Nersessian (2014) report on a 4-year ethnographic investigation of research practices in two labs where they also collaborated with practicing systems biologists to design supplementary courses for modellers and experimenters (see section 5). For more information on the project, please visit http://clic.gatech.edu.
} 
To appear in Systems Research and Behavioral Science, 2019, 36:5. Special issue: ISSS Yearbook.

dogmatic approach was also the best way to locate those loci of trouble that could spark a crisis in normal science and, in the end, lead to a scientific revolution (Andersen, 2000, 2001a, 2001b). ${ }^{14}$

On Kuhn's view, normal science within a discipline is therefore characterized by cognitive stability. This presents a challenge to interdisciplinary integration that requires cognitive flexibility. For scientists participating in interdisciplinary research it is therefore important to become aware of tacit assumptions of what constitutes good research practices in their respective fields (Nersessian, 2017). In contrast to what Kuhn found important for science education half a century ago, it is today important that science education prepares scientists for interdisciplinary collaboration by increasing students' epistemic awareness of their tacit assumptions. Awareness of the existence and implications of different disciplinary standards (Sections 2 and 3) can be facilitated through exercises where students are asked to identify hidden assumptions and discuss their implications. Such exercises can, for example, be based on a comparison of exemplars presented in textbooks or published scientific papers in various fields (Goddiksen, 2015). Similarly, identifying and comparing important exemplars in textbooks from different disciplines can be useful for scholars aiming to understand the background for some of the difficulties of interdisciplinary collaboration, as well as for educators aiming to teach interdisciplinary problem-solving (Goddiksen, 2013; 2015). Exercises can also involve comparisons of figures or examples in scientific publications where differences can also provide insights to the difficulties of understanding and combining different methodological and conceptual tools. ${ }^{15}$

Another approach has been developed by philosophers in the Toolbox Dialogue Initiative (http://toolboxproject.org/). Their approach is based on facilitated workshops that enable collaborators with different disciplinary backgrounds to engage in a structured dialogue about their research assumptions (Eigenbrode et al., 2007; O'Rourke \& Crowley, 2012). In this way, collaborators can gain the mutual understanding that is required for an effective collaboration. Similarly, Lindvig, Hillersdal and Earle (2017) have developed the gamified tool CoNavigator that enables interdisciplinary groups to visualize the interdisciplinary topography

\footnotetext{
${ }^{14}$ Kuhn therefore saw an essential tension between tradition and innovation in disciplinary science (Kuhn, 1977). Later, Andersen (2013) has argued that there is a second essential tension in interdisciplinary science between a discipline-based epistemic autonomy of the individual scientist and a discipline-crossing epistemic dependence between collaborating scientists.

15 In Denmark, a philosophy of science course is mandatory for all science students, and one exercise we often use is to ask students to characterize the elements of Kuhn's disciplinary matrix from the perspective of their field - and to compare these characteristics to other fields. Students enrolled in nanoscience programs in Aarhus and Copenhagen described a discrepancy between their physics classes and biology classes not unlike the differences outlined in Section 3 (see also Goddiksen, 2014b).
} 
To appear in Systems Research and Behavioral Science, 2019, 36:5. Special issue: ISSS Yearbook.

of their field, explore the possible connections between their areas of expertise, and demonstrate how their individual competences can be utilized in the collaboration.

Another challenge to interdisciplinary integration is related to the range of expertise required. Often, no single scientist will alone possess expertise in all fields involved in an interdisciplinary project. Collaborators therefore need to be able to integrate their various contributions, although they may not fully understand all details of each other's contributions. In this situation, they need to establish a "trading zone" (Galison, 1997) in which results and methods can be communicated and discussed. Again epistemic awareness of differences in the tacit assumptions of what constitute good research practices in different fields may be important for enabling such cross-disciplinary communication (Goddiksen, 2014b; MacLeod \& Nersessian, 2014). For instance, lack of familiarity with how experimental data are generated, and the associated challenges and uncertainties, can cause tensions between modellers and experimenters and lead to disagreements on the validity of the resulting models (MacLeod, 2018; MacLeod \& Nersessian, 2014).

Similarly, several scholars have commented upon the challenge of the varying degrees of mathematical training among researchers working in contemporary life sciences, which presents a practical challenge not only for interdisciplinary integration but also for communication and uptake of scientific results across different approaches. A study published in PNAS found that the density of equations in an article has significant negative impact on citation rates and that "heavy use of equations impedes communication among biologists" (Fawcett \& Higginson, 2012). The authors suggest that the examined papers could be made more accessible if difficult equations were explained better or moved to the appendix section. In response, others argued that "mathematical illiteracy impedes progress in biology" (Chitnis \& Smith, 2012; see also Gibbons, 2012). They argue that the increasing emphasis on complex systems and automated data production inevitably leads to changes in the mathematical and statistical skills needed to contribute to modern biology. Whether one agrees with the mathematically inclined scholar or not, the debate draws attention to the importance of addressing literacy requirements for contributory expertise in modern biology and for discussing how inter-subjectively shared frameworks of representational practices can be supported (Carusi, 2011; Carusi, Burrage, \& Rodríguez, 2012).

Another study suggests that educational background and skills for representational competence even affects measures at the perceptual level, i.e., that science students that are less confident in their math skills spends less time on the interpretation of information displayed in mathematical terms (Stieff, 
To appear in Systems Research and Behavioral Science, 2019, 36:5. Special issue: ISSS Yearbook.

Hegarty, \& Deslongchamps, 2011). ${ }^{16}$ Since the design, interpretation, and coordination of representations are central to interdisciplinary collaboration, empirical studies of how scientists process and use representational information may prove very useful for addressing challenges for cross- and interdisciplinary communication and integration. One way to deal with the present challenge may be to strengthen the mathematical literacy of experimental biologists. Again, what is required is not an education in mathematical modelling, but a modelling course sufficient to develop interactional expertise (MacLeod \& Nersessian, 2014).

An example of an initiative towards this aim is an introductory course in interdisciplinary problem-solving within systems biology, where a complex problem in biomedicine was designed to scaffold cognitive processes that required inputs from several disciplines (Voit, 2014; Voit, Newstetter, \& Kemp, 2012). Another option is to set up exchange programs between collaborative teams, so that modellers and experimenters get experience with the scientific practice in the "other camp" (Nersessian 2017). For instance, an introductory biosystems modelling course for bioscientists and novice modellers was developed in collaboration between philosophers of science and science faculty. Feedback from participants suggests that there can be great pay-offs even from short courses in modelling. A biologist interviewed after the course reports on how failures of communication could have been avoided had she taken the course earlier: "It would have been very helpful for me to understand what kind of data he needed; to understand what kinds of questions he should have been asking me" (MacLeod \& Nersessian, 2014, p. 237). The quote illustrates how an understanding of what kinds of data are needed for modelling is central to a fruitful collaboration between experimenters and modellers.

Modellers may also have to acquire more familiarity with the world of experimenters. Modellers often experience frustrations with experimenters for not being able to or willing to produce the kinds of data needed for the models. Part of the challenge results from separation of time-scales for running simulations and conducting lab experiments. Generating the data needed by modellers often takes a long time, and the particular type of data may be less interesting for the aims of experimental biologists, or may not be possible at all with current experimental techniques (MacLeod \& Nersessian, 2014). Despite publications describing the current situation as big data science, characterized by a "flood of data", research practices within systems biology are often constrained by the lack of good quality data that demand vast

\footnotetext{
${ }^{16}$ The study combined verbal protocols and eye fixation data to study how students coordinate and interpret domainspecific multi-representational displays.
} 
To appear in Systems Research and Behavioral Science, 2019, 36:5. Special issue: ISSS Yearbook.

experimental resources to create. ${ }^{17}$ That modellers understand experimental challenges is not only important for practical purposes of coordinating joint work but also for understanding the reliability (or uncertainty) of specific data sources and to take data constraints into account when developing and validating models (Carusi, 2014; MacLeod, 2018; Rowbottom, 2011). Accordingly, it is not only important that experimenters understand mathematical modelling, but also that modellers gain experience with the challenges and constraints of experimental practice.

Taking such issues into consideration as a result of empirical observations, MacLeod and Nersessian (2014) argue that a bimodal strategy where modellers also conduct experiments, and where experimenters also have some background in modelling, can provide a better coordination and integration of modelling and experimentation. ${ }^{18}$ Another, and perhaps less demanding, strategy is to design courses or summer school in experimental practices for modellers. MacLeod and Nersessian (2014) report on successful outcomes of such training in terms of what they call "meta-level cognition" and which is central to interactional expertise. After getting first-hand experience with experimentation, modellers report that they have gained increased understanding of the difficulties of experimental practices, and what kind of data is possible to generate. This awareness may help avoid frustrations resulting from modellers asking for data that would require years of experimental work to create, and which may not be worth the effort from experimenters' perspective (MacLeod, 2018). A better understanding of the needs (experimental data or models) of the collaborator, as well as the requirements and possibilities for producing these, can thus make more explicit the constraints that the collaboration must operate within.

A profound challenge facing interdisciplinary projects within systems sciences is, however, that there is a lack of fundamental theories to constrain and guide problem-solving activities. Since solving one problem does not necessarily prepare students or researchers for the next, Nersessian (2017) uses the term of an "adaptive problem space" to describe the relative unstructured learning and problem-solving environment characteristic of integrative systems biology. It remains to be seen whether this challenge can be addressed through the development of a unified theoretical framework of the systems sciences (Rousseau, 2017), or through more localized exemplars of useful problem-solving strategies.

\footnotetext{
${ }^{17}$ A lab director interviewed by MacLeod \& Nersessian (2014, p. 234) expressed the "data bottleneck" this way: "I've said it for 20 years, you need 10 experimentalists for every modeler".

${ }^{18}$ They also describe one disadvantage of the bimodal strategy as the potential risk of experimental biases if experimental data are produced to validate a model developed by the same researchers. On the other hand, they argue that bimodal modelers show greater interest in accounting for mechanistic details that may challenge the models (MacLeod \& Nersessian 2014, p. 236).
} 
To appear in Systems Research and Behavioral Science, 2019, 36:5. Special issue: ISSS Yearbook.

\section{Summary and concluding remarks}

Differences in disciplinary standards are often not explicitly visible in monodisciplinary settings. As scientists, or philosophers, we are often unaware of the norms and assumptions guiding our daily research practice because these constitute the very framework we think through or practice within. As argued in Section 2 , science students adopt such standards by learning exemplar cases and problem-solutions that exhibit valued characteristics of the given disciplines. An important point in Kuhn's philosophy is that it would be counterproductive for scientists to constantly question the elements and assumptions that make up a disciplinary paradigm or matrix, because these assumptions and standards are what enable scientific analysis. But disciplinary standards are important to address when these become visible as obstacles for collaboration, e.g., when we encounter different views on the appropriate methodology or research question. Accordingly, awareness of such standards and their implications are important for identifying problems associated with an inflexibility of the scientifically encoded mind, which may create blind spots and make us unable to engage in interdisciplinary collaborations in productive ways.

Differences in explanatory and methodological preferences may provide productive dialectical resistance to sharpen a field's identify as an impetus to the development of specialized areas and interdisciplinary innovation. However, at the level of local interdisciplinary collaboration, differences in scientific worldviews among experimenters and systems theoretical modellers are often perceived as a source of frustration. A view from philosophy of science and social science can shed light on the cognitive constraints of integrative practices which at the same time provide an opportunity for innovation and advanced problemsolving, and a potential obstacle for interdisciplinary integration. We have aimed to unpack some aspects of an inherent (second) essential tension in interdisciplinary practices (Andersen, 2013) - between domainspecific disciplinary traditions and interdisciplinary innovation. As documented in a number of cases, differences in philosophical viewpoints and expertise among collaborators can lead to challenges for integration and hindrance of epistemic trust among collaborators. We have focused on examples from systems biology, which is characterized by different opinions on what systems biology is and what count as a good model or explanation.

In systems biology, interdisciplinary problem-solving often occurs through a division of labour between experimental biologists and modelers trained in physics or engineering, which gives rise to debates on the relevant research questions and status of specific models and explanations. When researchers impose their own epistemic standards on other fields, the result is often that the attempts are met with criticism or 
To appear in Systems Research and Behavioral Science, 2019, 36:5. Special issue: ISSS Yearbook.

ignorance. An example is the tension between experimental biologists and researchers inspired by dynamical systems theory in stem cell biology, described in Section 3. In this field, explaining for the theoretically inclined researchers aim means to identify general laws that account for the dynamics of biological systems, whereas experimenters aim for causally anchored mechanistic explanations of molecular processes. Such differences often lead to provocative statements in scientific publications, e.g. that many of the abstract models are biologically irrelevant, or, from the theoretical perspective, that the qualitative mechanistic diagrams of experimenters are merely 'ad hoc metaphors' that fail to provide a mathematical understanding (Huang, 2011a, 2011b). Such tensions cannot be addressed by improving institutional and governance structures for research but require reflection upon philosophical values and standards underpinning different research practices.

Another set of challenges relate to gaps in the types and levels of expertise and familiarity with the theoretical and methodological frameworks of modelling and experimentation. We have described a set of challenges associated with gaps of expertise and pointed to ways in which some challenges may be overcome through courses that facilitate interactional expertise in the fields of collaborators. Some research problems may be best addressed through a bimodal strategy where researchers are able to do both experiments and modelling (MacLeod \& Nersessian, 2014). Other problems may require a very high level of expertise in both fields which makes this strategy unfeasible. However, even in these cases, an understanding of the worldviews and practical constraints of the collaborator's field may be central to establish a sufficient level of epistemic trust (Andersen, 2016), which is required in cases where it is not possible to critically examine the works of collaborators due to lack of expertise with the given methods. In this sense, the problem of interdisciplinary integration is also a problem of trust as it is developed and practices in scientific collaborations.

In summary, challenges for interdisciplinary collaboration provides a source of philosophical insights to ideals and standards operative in contemporary system sciences. But addressing and explicating the theoretical background for the different views may also help facilitate interdisciplinary collaboration and improve science education. A recognition of the values and standards guiding different approaches, as well as their respective aptitudes and limitations, can provide an improved understanding of complementary perspectives and suggest ways of bridging between these. Thus, interdisciplinary problem-solving - and the inherent tensions and challenges - open up new territories for productive interdisciplinary interactions also between scientists and philosophers. 


\section{References}

Abraham, T. H. (2004). Nicolas Rashevsky's mathematical biophysics. Journal of the History of Biology, 37(2), 333-385.

Aderem, A. S. (2005). Systems Biology: Its Practice and Challenges. Cell, 121(4), 511-513.

Alon, U. (2007). An introduction to systems biology: Design principles of biological circuits. Boca Raton: Chapman and Hall.

Andersen, H. (2000). Learning by Ostension: Thomas Kuhn on Science Education. Science \& Education, 9(9), 91-106.

Andersen, H. (2001a). The history of reductionism versus holistic approaches to scientific research. Endeavour, 25(4), 153-156.

Andersen, H. (2001b). On Kuhn. Belmont, CA: Wadsworth.

Andersen, H. (2013). The Second Essential Tension. Topoi, 32(1), 3-8.

Andersen, H. (2016). Collaboration, interdisciplinarity, and the epistemology of contemporary science. Studies in the History and Philosophy of Science Part A, 56, 1-10.

Apostel, L. (1972). Interdisciplinarity Problems of Teaching and Research in Universities. Paris: OECD.

Bechtel, W. (1986). Integrating Scientific Disciplines. Dordrecht: Springer.

Bechtel, W., \& Richardson, R. C. (1993). Discovering Complexity: Decomposition and Localization as Strategies in Scientific Research. Princeton, New Jersey: Princeton University Press.

Braillard, P.-A. (2010). Systems Biology and the Mechanistic Framework. History and Philosophy of the Life Sciences, 32, 43-62.

Braillard, P.-A., \& Malaterre, C. (2015). Explanation in Biology: Springer.

Brigandt, I. (2013). Systems biology and the integration of mechanistic explanation and mathematical explanation. Studies in History and Philosophy of Biological and Biomedical Sciences, 44(4), 477-492.

Brigandt, I. (2015). From developmental constraint to evolvability: how concepts figure in explanation and disciplinary identity. In A. Love (Ed.), Conceptual Change in Biology (pp. 305-325). Amsterdam: Springer.

Brigandt, I., \& Love, A. C. (2017). Reductionism in biology. Stanford Encyclopedia of Philosophy (Avaible at: https://plato.stanford.edu/entries/reduction-biology/).

Brigandt, I., \& Love', A. C. (2012). Conceptualizing evolutionary novelty: Moving beyond definitional debates. Journal of Experimental Zoology Part B: Molecular and Developmental Evolution, 318, 417-427.

Brush, S. G. (2000). Thomas Kuhn as historian of science. Science and Education(9), 39-58.

Burian, R., Richardson, R. C., \& Van der Steen, W. (1996). Against Generality: Meaning in Genetics and Philosophy. Studies in History and Philosophy of Science, 27(1), 1-29.

Calvert, J. (2010). Systems Biology, Interdisciplinarity and Disciplinary Identity. In J. N. Parker, N. Vermeulen, \& B. Penders (Eds.), Collaboration in the new life sciences (pp. 219-244). London: Ashgate.

Calvert, J., \& Fujimura, J. H. (2011). Calculating life? Duelling discourses in interdisciplinary systems biology. When Physics Meets Biology, 42(2), 155-163.

Campbell, N. A., \& Reece, J. B. (2005). Biology. San Francisco, CA: Pearson. Education.

Carusi, A. (2011). Computational Biology and the Limits of Shared Vision. Perspectives on Science, 19(3), 300-335. 
Carusi, A. (2014). Validation and variability: Dual challenges on the path from systems biology to systems medicine. Studies in History and Philosophy of Biological and Biomedical Sciences, 48, 28-37.

Carusi, A., Burrage, K., \& Rodríguez, B. (2012). Bridging experiments, models and simulations: an integrative approach to validation in computational cardiac electrophysiology. American Journal of Physiology - Heart and Circulatory Physiology, 303(2), H144-H155.

Chang, H. (2015). Reductionism and the relation between chemistry and physics. In T. Arabatzis, J. Renn, \& A. Simoes (Eds.), Relocating the History of Science (pp. 193-209). Cham, Switzerland: Springer.

Chitnis, N., \& Smith, T. A. (2012). Mathematical illiteracy impedes progress in biology. Proceedings of the National Academy of Sciences, 109(45), E3055-E3055.

Collins, H. M., \& Evans, R. (2002). The third wave of science studies: Studies of expertise and experience. Social Studies of Science, 32, 235-296.

Craver, C. (2007). Explaining the Brain, Mechanisms and the Mosaic Unity of Neuroscience. Oxford: Clarendon Press.

Crow, M. M., \& Dabars, W. B. (2015). Designing the new American university. Baltimore: John Hopkins University Press.

Darden, L., \& Maull, N. (1977). Interfield theories. Philosophy of Science, 44, 43-64. doi:pmid:

Drack, M., \& Wolkenhauer, O. (2011). System approaches of Weiss and Bertalanffy and their relevance for systems biology today. Seminars in Cancer Biology, 21(3), 150-155.

Doyle, J. (2001). Beyond the spherical cow. Nature, 411(10), 151-152.

Dupré, J. (1994). Against scientific imperialism. Proceedings of the Biennial Meeting of the Philosophy of Science Association, 1994(2), 374-381.

Eigenbrode, S. D., O'Rourke, M., Wulfhorst, J. D., Althoff, D. M., Goldberg, C. S., Merrill, K., . . . Winowiecki, L. (2007). Employing philosophical dialogue in collaborative science. Bioscience, 57(1), 55-64.

Fagan, M. B. (2010). Stems and standards: social interaction in the search for blood stem cells. Journal of the History of Biology, 43(1), 67-109.

Fagan, M. B. (2012). Waddington redux: models and explanation in stem cell and systems biology. Biology \& Philosophy, 27(2), 179-213.

Fagan, M. B. (2016). Stem cells and systems models: Clashing views of explanation. Synthese, 193, 873-907.

Fagan, M. B. (2017). Interdisciplinarity, philosohy and systems biology. In S. Green (Ed.), Philosophy of systems biology. Perspectives from scientists and philosophers (pp. 87-97). Cham, Switzerland: Springer International Publishing.

Fawcett, T. W., \& Higginson, A. D. (2012). Heavy use of equations impedes communication among biologists. Proceedings of the National Academy of Sciences, 109(29), 11735-11739.

Furusawa, C., \& Kaneko, K. (2012). A dynamical-systems view of stem cell biology. Science, 338(6104), 215-217.

Galison, P. (1997). Image \& logic: A material culture of microphysics. Chicago: The University of Chicago Press.

Gibbons, J. (2012). Do not throw equations out with the theory bathwater. Proceedings of the National Academy of Sciences of the USA, 109(45), E3054.

Goddiksen, M. (2013). Interdisciplinarity and explanation: How interdisciplinary education calls for a new approach to research on scientific explanations. Proceedings of the 12th Biennial 
To appear in Systems Research and Behavioral Science, 2019, 36:5. Special issue: ISSS Yearbook.

International History, Philosophy, and Science Teaching Group Conference, 12 (Available: http://archive.ihpst.net/2013-pittsburgh/conference-proceedings/).

Goddiksen, M. (2014a). Clarifying interactional and contributory expertise. Studies In History and Philosophy of Science Part A, 47, 111-117.

Goddiksen, M. (2014b). Philosophical Perspectives on Interdisciplinary Science Education: Characterizing important expertises through a practice oriented analysis of integration and explanation. Aarhus University.

Goddiksen, M. (2015). An empirical method for the study of exemplar explanations. In S. Wagenknecht, N. J. Nersessian, \& H. Andersen (Eds.), Empirical Philosophy of Science (pp. 105-126). Cham, Switzerland: Springer.

Goddiksen, M. (2017). The theory of interdisciplinarity: An introduction for educators. In J. Dolin \& C. Holm (Eds.), Interdisciplinary Education at the University of Copenhagen (pp. 1-16).

Copenhagen: University of Copenhagen. Available:

http://www.ind.ku.dk/english/interdisciplinarity/materials/the-theory-ofinterdisciplinarity-mpg-2017/The Theory of Interdisciplinarity-MPG 2017.pdf.

Green, S. (2015a). Can biological complexity be reverse engineered? Studies in History and Philosophy of Biological and Biomedical Sciences, 53, 73-83.

Green, S. (2015b). Revisiting Generality in the Life Sciences: Systems Biology and the Quest for General Principles. Biology and Philosophy, 30, 629-652.

Green, S. (2017). Philosophy of Systems and Synthetic Biology. Stanford Encyclopedia of Philosophy, https://plato.stanford.edu/entries/systems-synthetic-biology/.

Green, S., Fagan, M., \& Jaeger, J. (2015). Explanatory Integration Challenges in Evolutionary Systems Biology. Biological Theory, 10(1), 18-35. doi:10.1007/s13752-014-0185-8.

Green, S., \& Jones, N. (2016). Constraint-based reasoning for search and explanation. Dialectica, 70(3), 343-374.

Green, S., \& Wolkenhauer, O. (2013). Tracing organizing principles: Learning from the history of systems biology. History and Philosophy of the Life Sciences, 35(4), 555-578.

Griesemer, J. (2013). Integration of approaches in David Wake's model-taxon research platform for evolutionary morphology. Studies in History and Philosophy of Science Part C: Studies in History and Philosophy of Biological and Biomedical Sciences, 44(4), 525-536.

Gross, F. (2017). Towards a methodology for systems biology. In S. Green (Ed.), Philosophy of systems biology. Perspectives from scientists and philosophers (pp. 109-116). Cham, Switzerland: Springer International Publishing.

Gross, F., \& Green, S. (2017). The sum of the parts: Large-scale modeling in systems biology. Philosophy, Theory and Practice in Biology, 9.

Hempel, C. (1966). Philosophy of Natural Science. Englewod Cliffs, N-J.: Prentice-Hall.

Hempel, C., \& Oppenheim, P. (1948). Studies in the Logic of Explanation. Philosophy of Science, 15(2), 135-175.

Hofmeyr, J.-H. S. (2017). Exploring the metabolic marketplace through the lens of systems biology. In S. Green (Ed.), Philosophy of systems biology. Perspectives from scientists and philosophers (pp. 117-124). Cham, Switzerland: Springer International Publishing.

Hood, L., \& Flores, M. (2012). A personal view on systems medicine and the emergence of proactive P4 medicine: predictive, preventive, personalized and participatory. New biotechnology, 29(6), 613-624. 
To appear in Systems Research and Behavioral Science, 2019, 36:5. Special issue: ISSS Yearbook.

Huang, S. (2009). Reprogramming cell fates: reconciling rarity with robustness. BioEssays, 31(5), 546-560.

Huang, S. (2011a). The molecular and mathematical basis of Waddington's epigenetic landscape: A framework for post-Darwinian biology? BioEssays, 34(2), 149-157.

Huang, S. (2011b). Systems biology of stem cells: three useful perspectives to help overcome the paradigm of linear pathways. Philosophical Transactions of the Royal Society B: Biological Sciences, 366(1575), 2247-2259.

Isalan, M., Lemerle, C., Michalodimitrakis, K., Horn, C., Beltrao, P., Raineri, E., . . Serrano, L. (2008). Evolvability and hierarchy in rewired bacterial gene networks. Nature, 452(7189), 840-845.

Joaquim, L. M., Freire, O., \& El-Hani, C. N. (2019). From physics to biology: physicists in the search for systemic biological explanations. European Journal of Philosophy of Science, 9(30), https://doi.org/10.1007/s13194-019-0254-8.

Kaneko, K. (2011). Characterization of stem cells and cancer cells on the basis of gene expression profile stability, plasticity, and robustness. BioEssays, 33(6), 403-413.

Kitano, H. (2005). Scientific and technical challenges for systems biology. In H. V. Westerhoff \& L. Alberghina (Eds.), Systems Biology. Definitions and Perspectives (pp. 373-383): Springer.

Kjeldsen, T. H. (2017). An early debate in mathematical biology and its value for teaching: Rashevsky's 1934 paper on cell division. The Mathematical Intelligencer, 39(2), 36-45.

Klein, J. (2010). A taxonomy of interdisciplinarity. In R. Frodeman, J. T. Klein, \& C. Mitcham (Eds.), The Oxford Handbook of Interidisciplinarity (pp. 15-30). Oxford: Oxford University Press.

Kolodkin, A., \& Westerhoff, H. (2011). Parsimony for systems biology: Shaving Occam's razor away. Eur. Commun. Math. Theor. Biol, 14, 149-152.

Kuhn, T. S. (1962/1996). The Structure of Scientific Revolutions (3rd ed.). Chicago: University of Chicago Press.

Kuhn, T. S. (1977). The Essential Tension. Chicago: University of Chicago Press.

Lattuca, L. R. (2001). Creating interdisciplinarity: Interdisciplinary research and teaching among college and university faculty. Nashville, Tenesse: Vanderbilt University Press.

Lazebnik, Y. (2002). Can a biologist fix a radio?-Or, what I learned while studying apoptosis. Cancer Cell, 2(3), 179-182.

Leonelli, S. (2007). What Is in a Model? Combining Theoretical and Material Models to Develop Intelligible Theories. In M. D. Laubichler \& G. B. Müller (Eds.), Modeling Biology: Structure, Behaviors, Evolution (pp. 15-35). Cambridge, MA: The MIT Press.

Leonelli, S. (2008). Bio-ontologies as tools for integration in biology. Biological Theory, 3(1), 7.

Lindvig, K., Hillersdal, L., \& Earle, D. (2017). Interdisciplinary Tool Helps Fast-Track Interdisciplinary Learning and Collaboration. Integrative Pathways, 39(2), 171-174.

Machamer, P., Darden, L., \& Craver, C. (2000). Thinking about mechanisms. Philosophy of Science, 67(1), 1-25. doi:pmid:

MacLeod, M. (2018). What makes interdisciplinarity difficult? Some consequences of domain specificity in interdisciplinary practice. Synthese, 195(2), 697-720.

MacLeod, M., \& Nersessian, N. J. (2014). Strategies for coordinating experimentation and modeling in integrative systems biology. Journal of Experimental Zoology (Molecular and Developmental Evolution), 9999, 1-10.

Mäki, U. (2013). Scientific imperialism: Difficulties in definition, identification, and assessment. International Studies in the Philosophy of Science, 27(3), 325-339. 
Matthiessen, D. (2017). Mechanistic Explanation in Systems Biology: Cellular Networks. The British Journal for the Philosophy of Science, 68(1), 1-25.

Mayr, E. (2005). What Makes Biology Unique? Considerations on the Autonomy of a Scientific Discipline. Cambridge UP: Cambridge University Press.

Mitchell, S. (2002). Integrative Pluralism. Biology and Philosophy, 17, 55-70-55-70.

Mitchell, S. (2005). Biological Complexity and Integrative Pluralism. Cambridge: Cambridge University Press.

Nersessian, N. J. (2017). Systems biology modeling practices: reflections of a philosopherethnographer. In S. Green (Ed.), Philosophy of systems biology. Perspectives from scientists and philosophers (pp. 215-226). Cham, Switzerland: Springer International Publishing.

O'Malley, M., \& Dupré, J. (2005). Fundamental issues in systems biology. BioEssays, 27(12), 12701276.

O'Malley, M., \& Boucher, Y. (2005). Paradigm change in evolutionary microbiology. Studies in History and Philosophy of Science Part C: Studies in History and Philosophy of Biological and Biomedical Sciences, 36(1), 183-208.

O'Malley, M. A., \& Soyer, O. S. (2012). The roles of integration in molecular systems biology. Studies in History and Philosophy of Biological and Biomedical Sciences, 43(1), 58-68.

O'Rourke, M., \& Crowley, S. J. (2012). Philosophical intervention and cross-disciplinary science: the story of the Toolbox Project. Synthese, 190(11), 1-18.

Oppenheim, P., \& Putnam, H. (1958). The unity of science as a working hypothesis. In H. Feigl, M. Scriven, \& G. Maxwell (Eds.), Concepts, theories, and the mind-body. problem (pp. 3-36). Minneapolis: University of Minnesota Press.

Petrie, H. G. (1976). Do you see what I see? The epistemology of interdisciplinary inquiry. Educational Researcher, 5, 9-15.

Rashevsky, N. (1935). Mathematical Biophysics. Nature, 135, 528-530.

Rashevsky, N. (1938a). Mathematical biophysics: Physicomathematical foundations of biology. Chicago: University of Chicago Press.

Rashevsky, N. (1938b). The Relation of Mathematical Biophysics to Experimental Biology. Acta Biotheoretica, 4(2).

Rousseau, D. (2017). Systems research and the quest for scientific systems principles. Systems, $5(2), 25$.

Rousseau, D., Wilby, J., Billingham, J., \& Blachfellner, S. (2016). A typology for the systems field. Systema: connecting matter, life, culture and technology, 4(1), 15-47.

Rowbottom, D. P. (2009). Models in Biology and Physics: What's the Difference? Foundations of Science, 14(4), 281-294. doi:10.1007/s10699-009-9160-4 pmid:

Rowbottom, D. P. (2011). Approximations, idealizations and 'experiments' at the physics-biology interface. Studies in History and Philosophy of Biological and Biomedical Sciences, 42(2), 145-154.

Sá, C. M. (2008). 'Interdisciplinary strategies' in US research universities. Higher Education, 55(5), 537-552.

Shmailov, M. M. (2016). Intellectual Pursuits of Nicolas Rashevsky. Cham, Switzerland: Springer International Publishing.

Smith, K. C. (1992). Neo-rationalism versus neo-Darwinism: Integrating development and evolution. Biology and Philosophy, 7(4), 431-451. 
Star, L., \& Griesemer, J. (1989). Institutional ecology, "translations," and boundary objects. In M. Biagiolo (Ed.), The science studies reader (pp. 505-524). New York: Routledge.

Stieff, M., Hegarty, M., \& Deslongchamps, G. (2011). Identifying representational competence with multi-representational displays. Cognition and Instruction, 29(1), 123-145.

Voit, E. O. (2014). Mesoscopic modeling as a starting point for computational analyses of cystic fibrosis as a systematic disease. Biochimica et Biophysica Acta. (BBA)-Proteins and Proteomics, 1844, 258-270.

Voit, E. O. (2017). From a fascination with arrow diagrams to witnessing a tipping point in biology. In S. Green (Ed.), Philosophy of systems biology. Perspectives from scientists and philosophers (pp. 247-256). Cham, Switzerland: Springer International Publishing.

Voit, E. O., Newstetter, W. C., \& Kemp, M. L. (2012). A feel for systems. Molecular Systems Biology, $8,609$.

Weingart, P. (2000). Interdisciplinarity: The Paradoxical Discourse. In P. Weingart \& N. Stehr (Eds.), Practising Interdisciplinarity (pp. 40-40): University of Toronto Press.

Wiengart, P., \& Padberg, B. (Eds.). (2014). University Experiments in Interdisciplinarity. Obstacles and Opportunities. Bielefeld: Transcript Verlag.

Wolkenhauer, O. (2001). Systems biology: The reincarnation of systems theory applied in biology. Briefings in bioinformatics, 2(3), 258-270.

Wolkenhauer, O. (2017). From microscopes to macroscopes: Advancing biomedical research through systems approaches. In S. Green (Ed.), Philosophy of systems biology. Perspectives from scientists and philosophers (pp. 257-265). Cham, Switzerland: Springer International Publishing. 